\title{
Nutzung medizinischer Schreibservices - eine datenschutzrechtliche Sicht
}

\author{
Medizinische Schreibservices bieten das Transkribieren ärztlicher Diktate wie Kranken- \\ kassenberichte oder Gutachten an. Es stellt sich die Frage, ob dies eine Verletzung \\ der ärztlichen Schweigepflicht darstellt.
}

\section{Ursula Uttinger ${ }^{a}$,}

Michael Liebrenz ${ }^{b}$

a lic. iur.; exec. MBA HSG, Geschäftsführerin Activita Care Management AG Präsidentin DatenschutzForum Schweiz, Dozentin für Datenschutz an diversen Hochschulen

b Dr. med., Facharzt für Psychiatrie und Psychotherapie, Research Fellow Division of Law, Ethics, and Psychiatry Columbia University New York (USA), Leiter Gutachtenstelle für Zivil- und Öffentlichrechtliche Fragestellungen, Psychiatrische Universitätsklinik Zürich

\footnotetext{
*ine ausführliche Fassung dieses Beitrags und die Literaturangaben finden sich unter www.saez.ch

$\rightarrow$ Aktuelle Ausgabe oder $\rightarrow$ Archiv $\rightarrow 2014 \rightarrow 46$.
}

Korrespondenz:

Dr. med. Michael Liebrenz

Psychiatrische Universitätsklinik Militärstrasse 8

CH-8021 Zürich

michael.liebrenz[at]uzh.ch

\section{Einleitung}

In Anbetracht steigender Kosten im Gesundheitssystem wird der Ärzteschaft von Ökonomen eine Konzentration auf sogenannte «Primär- und Sekundärprozesse», d.h. all jene Leistungen, die der Genesung des Patienten dienen (Diagnostik/Therapie), empfohlen $[1,2,6]^{*}$. Hingegen soll die Auslagerung von «Tertiärprozessen» - im kaufmännischen Bereich sind dies u.a. Leistungsabrechnung, Patientenadministration und Sekretariatsdienste - geprüft werden [6].

An dieser Stelle setzen zunehmend externe Dienstleister an [3-6]. Medizinische Schreibbüros oder Schreibservices bieten disziplinenübergreifend das Transkribieren von ärztlichen Diktaten (z.B. Krankenkassenberichten, Gutachten) an. Kostensenkung, Kundenorientierung, Qualität und schnelle «Turn around»-Zeiten gelten dabei als Vorteile des Outsourcings von Schreibdienstleistungen an spezialisierte Anbieter [7]. Vor dem Hintergrund dieser Entwicklung stellt sich eine Reihe von Fragen, deren zentralste diejenige ist, ob die Nutzung von Schreibservices eine Verletzung der ärztlichen Schweigepflicht darstellt.

Die ärztliche Schweigepflicht ist dabei als berufliche Schweigepflicht in Art. 321 StGB [8] geregelt. Das Berufsgeheimnis umfasst nicht einzig die ausdrücklich angeführten Berufe, sondern auch «ihre Hilfspersonen».

Als Hilfspersonen versteht man Personen, die den Geheimnisträger unterstützen (inklusive untergeordnete Hilfskräfte), sofern diese auch mit vertraulichen Informationen in Kontakt kommen [9]. Diese Hilfspersonen unterliegen den gleichen Schweigepflichten, müssen aber unter der «Leitung und Aufsicht» des Geheimnisträgers tätig sein. Im Sinne von Art. 321 StGB kann der Kreis der Hilfspersonen sehr weit gezogen werden, wobei es vor allem auf die Aufgabe und nicht auf die offizielle Funktion ankommt [10].

Eine Arztsekretärin in einem Spital oder in einer Praxis ist eine Hilfsperson im Sinne von Art. 101 OR [11], d.h. sie unterstützt bei der Erfüllung der Tätigkeit (Erfüllungsgehilfe) [12, 13]. Im Unterschied zur Hilfsperson wird beim Outsourcing eine Tätigkeit durch einen Dienstleister selbständig vorgenommen -

\section{Externalisation des tâches} administratives et protection

\section{des données}

Dans le milieu médical, de plus en plus de tâches administratives sont fournies par des prestataires externes. La loi sur la protection des données autorise le traitement de données par des tiers, à condition que cela soit fait dans le respect des principes généraux de protection des données. Mais en médecine, la loi sur la protection des données n'est pas le seul élément dont il faut tenir compte, le secret médical revêt en effet également un rôle central y compris pour le personnel auxiliaire. Si l'externalisation est en principe possible, elle doit être soumise à une règlementation claire et transparente entre les personnes impliquées.

meist auch längerfristig und in eigenen Räumlichkeiten [14].

Aus datenschutzrechtlicher Sicht ist ein Outsourcing eine Datenbearbeitung durch Dritte, die in Art. 10a DSG geregelt ist. Grundsätzlich ist eine solche möglich, sofern keine gesetzliche oder vertragliche Geheimhaltungspflicht dem entgegensteht [15]. Ein Outsourcing ist auch bei einer beruflichen Schweigepflicht möglich, sofern der Outsourcingnehmer zu einer entsprechenden Geheimhaltung verpflichtet wird [16, 17].

Werden die Daten durch einen Dritten bearbeitet, bleibt die Verantwortung für die Datenbearbeitung beim Auftraggeber. Dieser sollte die den Umständen angemessenen Sorgfaltspflichten anwenden. Neben der sorgfältigen Auswahl ist er auch für eine klare Instruktion verantwortlich. Zudem hat er sich zu vergewissern, dass die Datensicherheit gewährleistet wird.

Bei einer grenzüberschreitenden Datenbearbeitung ist zu unterscheiden zwischen Ländern mit 


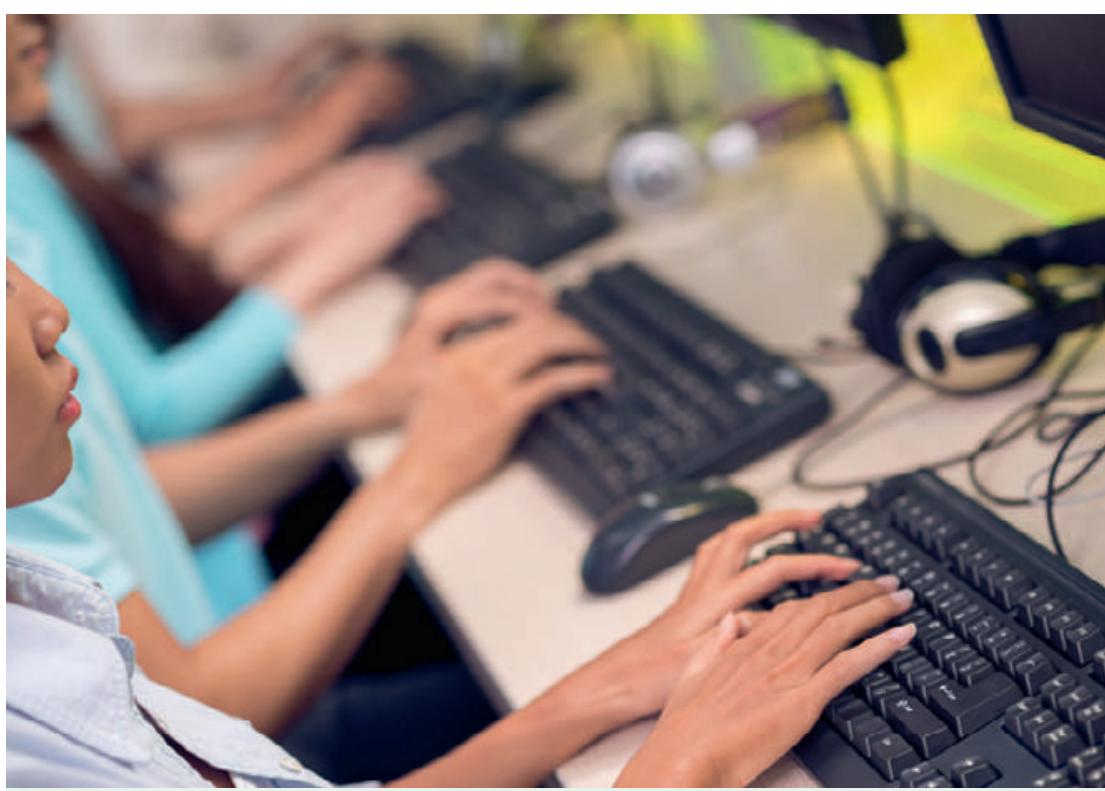

Grundlegend beim Outsourcing ärztlicher Schreibarbeiten: Der Ausführende muss entsprechend der Schweigepflicht zu Geheimhaltung verpflichtet werden. haltung der Verpflichtungen überprüfen zu können [23].

Der Arzt bleibt für das Tun seiner Hilfsperson verantwortlich, egal ob die Person in der Schweiz oder in einem anderen Land arbeitet. Die ärztliche Schweigepflicht ist dabei grundsätzlich weltweit verbreitet und wird häufig ähnlich der schweizerischen Gesetzgebung eigenständig sanktioniert [24-29]. Dies gilt unabhängig von den datenschutzrechtlichen Pflichten, deren Verletzung teilweise mit härteren Sanktionsandrohungen versehen sind als in der Schweiz.

Eine wichtige Rolle spielt zudem eine grösstmögliche Transparenz [30]. Grundsätzlich genügt eine stillschweigende oder konkludente Einwilligung [31]. Wichtig ist, dass die Einwilligung auf einer freien Entscheidfindung beruht, die betroffene Person genügend Informationen im Konkreten hat [32]. Werden Dritte involviert, sollte man die betroffenen Personen mindestens informieren, so dass sich diese durch Widerspruch dem Outsourcing widersetzen könnten. Aus Beweisgründen empfiehlt sich eine schriftliche Einwilligung.

einem gleichwertigen Datenschutzniveau und anderen. Die EU hat einen gleichwertigen Datenschutz; allerdings verfügen auch Staaten wie Uruguay oder Israel über einen solchen [18]. Der eidg. Datenschutz- und Öffentlichkeitsbeauftragte (EDÖB) ist gemäss Art. 31 Abs. 1 lit. d DSG [19] verpflichtet, eine Liste der Länder mit gleichwertigem Datenschutz zu führen. Diese ist auf der Homepage des EDÖB hinterlegt.

Ist das Land nicht auf der Liste des EDÖB, müssen zusätzliche Sicherheitsmassnahmen umgesetzt werden [20]. Bei einem Schreibservice drängt sich eine vertragliche Lösung auf [21]. Dazu gibt es verschiedene Musterverträge [22].

Es spielt keine Rolle, ob der Anbieter der Schreibdienste ein Büro in der Schweiz hat oder direkt im Ausland domiziliert ist. Entscheidend ist, wo die Datenbearbeitung erfolgt. Deshalb sollte beim Outsourcing vertraglich klar festgehalten werden, wo die Datenbearbeitung erfolgt und welche Rechte der Datenexporteur hat, um die Ein-

\section{Der Outsourcingnehmer muss zu einer entsprechenden Geheim- haltung verpflichtet werden.}

\section{Fazit}

Im medizinischen Umfeld werden vermehrt administrative Aufgaben durch externe Dienstleister erbracht. Das Datenschutzgesetz kennt eine Datenbearbeitung durch Dritte - unter Berücksichtigung der allgemeinen Datenschutzgrundsätze. Doch nicht nur das Datenschutzgesetz ist zu beachten, denn im medizinischen Umfeld spielt die berufliche Schweigepflicht, die auch Hilfspersonen umfasst, eine wichtige Rolle. Bei einer klaren Regelung und Transparenz gegenüber den betroffenen Personen ist ein Outsourcing - auch ins Ausland - grundsätzlich möglich. 


\section{Références}

1 Lundsgard-Hansen N, Stettler F. Herausforderungen im Schweizer Spitalwesen. In Book Herausforderungen im Schweizer Spitalwesen. PricewaterhouseCoopers; 2006.

2 Schüpfer G, Babst R. Risiken und Nebenwirkungen von Sparprogrammen auf die stationäre Medizin in der Schweiz. Praxis. (2002 to 2006). 2005;94:110311.

3 Lawler FH, Scheid DC, Viviani NJ: The cost of medical dictation transcription at an academic family practice center. Arch Fam Med. 1998;7:26972.

4 Hinickle J. RFP: Outsourcing Medical Transcription Perspectives 1995. Health Professions Institute. Fall 199.:4.

5 Kshetri N, Dholakia N. Offshoring of healthcare services: the case of US-India trade in medical transcription services. J Health Organ Manag. 2011; 25:94-107.

6 Ghodeswar B, Vaidyanathan J.Business process outsourcing: an approach to gain access to world-class capabilities. Business Process Management Journal. 2008; $14: 23-38$

7 Bikam J, Whiting S. Medical transcription outsourcing greased lightning? Healthcare financial management: journal of the Healthcare Financial Management Association. 2007;61:94-7.

8 SR 311.0: Art. 321 StGB Verletzung des Berufsgeheimnisses 1. Geistliche, Rechtsanwälte, Verteidiger, Notare, Patentanwälte, nach Obligationenrecht zur Verschwiegenheit verpflichtete Revisoren, Ärzte, Zahnärzte, Chiropraktoren, Apotheker, Hebammen, Psychologen sowie ihre Hilfspersonen, die ein Geheimnis offenbaren, das ihnen infolge ihres Berufes anvertraut worden ist oder das sie in dessen Ausübung wahrgenommen haben, werden, auf Antrag, mit Freiheitsstrafe bis zu drei Jahren oder Geldstrafe bestraft.

9 Trechsel S, Vest H. In Trechsel Stefan/Pieth Mark (Hrsg). Schweizersiches Strafgesetzbuch, Praxiskommentar. 2. Aufl. Zürich/St. Gallen; 2012, Art, 321 N13.

10 Oberholzer N. In: Niggli Marcel Alexander/Wiprächtiger Hans (Hrsg.) Basler Kommentar, Strafrecht II, Art. 111 392 StGB, 3. Auflage, Basel 2013, Art, 321 N 10.

11 SR 220: Art. 101 OR Haftung für Hilfspersonen 1 Wer die Erfüllung einer Schuldpflicht oder die Ausübung eines Rechtes aus einem Schuldverhältnis, wenn auch befugterweise, durch eine Hilfsperson, wie Hausgenossen oder Arbeitnehmer vornehmen lässt, hat dem andern den Schaden zu ersetzen, den die Hilfsperson in Ausübung ihrer Verrichtungen verursacht.

12 Vollenweider AC. Die Haftpflicht für medizinische Eingriffe, namentlich von Hilfspersonen, Rechtsgutachten gdk, 2001, Ziff. 2.2.2

13 Vgl. auch Schweizerische Gesellschaft der Vertrauensund Versicherungsärzte (SGV), Empfehlung Datenschutz Anhang 1, Mustervertrag Hilfsperson VA und VAD.

14 Vgl. auch Rundschreiben 2008/7 «Outsourcing Banken», Ziff. $1 \mathrm{f}$.

15 SR 235.1: Art. 10a DSG Datenbearbeitung durch Dritte

1 Das Bearbeiten von Personendaten kann durch Vereinbarung oder Gesetz Dritten übertragen werden, wenn:

a. die Daten nur so bearbeitet werden, wie der Auftraggeber selbst es tun dürfte; und

b. keine gesetzliche oder vertragliche Geheimhaltungspflicht es verbietet.

16 Rosenthal D. In: Rosenthal D, Jöhri Y (Hrsg). Handkommentar zum Datenschutzgesetz, Art. 10a N107

17 Widmer U. Rechtliche Rahmenbedingungen für das Outsourcing im Spitalbereich - Weitergabe von Patientendaten. In: datamaster September 2007, S. 20.

18 Vgl. Staatenliste des Eidg. Datenschutz- und Öffentlichkeitsbeauftragten (Stand des Datenschutzes weltweit)
19 SR 235.1 Art. 29 Abs. 1 DSG Der Beauftragte hat insbesondere folgende weiteren Aufgaben:

d. Er begutachtet, inwieweit die Datenschutzgesetzgebung im Ausland einen angemessenen Schutz gewährleistet.

20 Merkblatt Cloud Computing, Datenschutzbeauftragter Kanton Zürich, 2012, Ziff. 4.4

21 SR 235.1 DSG Art. 6 Grenzüberschreitende Bekanntgabe

2 Fehlt eine Gesetzgebung, die einen angemessenen Schutz gewährleistet, so können Personendaten ins Ausland nur bekannt gegeben werden, wenn:

a. hinreichende Garantien, insbesondere durch Vertrag, einen angemessenen Schutz im Ausland gewährleisten; b. die betroffene Person im Einzelfall eingewilligt hat;

22 Mustervertrag für das Outsourcing für Datenbearbeitung im Ausland des Eidg. Datenschutz- und Öffentlichkeitsbeauftragten, Modal Contracts for the transfer of personal data to third countries des Europarates.

23 Rosenthal D.(Fn 29), Art. 6 N40.

$24 \S 203$ StGB Verletzung von Privatgeheimnissen 1) Wer unbefugt ein fremdes Geheimnis, namentlich ein zum persönlichen Lebensbereich gehörendes Geheimnis oder ein Betriebs- oder Geschäftsgeheimnis, offenbart, das ihm als 1. Arzt, Zahnarzt, Tierarzt, Apotheker oder Angehörigen eines anderen Heilberufs, der für die Berufsausübung oder die Führung der Berufsbezeichnung eine staatlich geregelte Ausbildung anvertraut worden oder sonst bekanntgeworden ist, wird mit Freiheitsstrafe bis zu einem Jahr oder mit Geldstrafe bestraft.

$25 \S 121$ StGB Verletzung von Berufsgeheimnissen (1) Wer ein Geheimnis offenbart oder verwertet, das den Gesundheitszustand einer Person betrifft und das ihm bei berufsmässiger Ausübung eines gesetzlich geregelten Gesundheitsberufes oder bei berufsmässiger Beschäftigung mit Aufgaben der Verwaltung einer Krankenanstalt oder eines anderen Gesundheitsdiensteanbieters (§ 2 Z 2 des Gesundheitstelematikgesetzes 2012, BGBl. I Nr. 111/2012) oder mit Aufgaben der Kranken-, der Unfall-, der Lebens- oder der Sozialversicherung ausschliesslich kraft seines Berufes anvertraut worden oder zugänglich geworden ist und dessen Offenbarung oder Verwertung geeignet ist, ein berechtigtes Interesse der Person zu verletzen, die seine Tätigkeit in Anspruch genommen hat oder für die sie in Anspruch genommen worden ist, ist mit Freiheitsstrafe bis zu sechs Monaten oder mit Geldstrafe bis zu 360 Tagessätzen zu bestrafen.

26 Article 226-13 Code pénal:

La révélation d'une information à caractère secret par une personne qui en est dépositaire soit par état ou par profession, soit en raison d'une fonction ou d'une mission temporaire, est punie d'un an d'emprisonnement et de 15000 euros d'amende.

27 http://en.wikipedia.org/wiki/Legal_professional_ privilege (besucht am 9.7.2014).

28 Le professionnel doit respecter le secret de tout renseignement de nature confidentielle qui vient à sa connaissance dans l'exercice de sa profession. www2.publicationsduquebec.gouv.qc.ca/dynamicSearch/telecharge. php?type=2\&file=/C_26/C26.htm besucht am 9.7.2014

29 Art. 622 codice penale - Rivelazione di segreto professionale Chiunque, avendo notizia, per ragione del proprio stato o ufficio, o della propria professione o arte, di un segreto, lo rivela, senza giusta causa, ovvero lo impiega a proprio o altrui profitto, è punito, se dal fatto può derivare nocumento, con la reclusione fino a un anno o con la multa da euro 30 a euro 516.

30 Rechtliche Grundlagen im medizinischen Alltag - ein Leitfaden für die Praxis. Hrsg. Schweizerische Akademie für Medizinische Wissenschaften und der Verbindung der Schweizer Ärzte und Ärztinnen FMH, 2. Auflage, 2012, S. $99 \mathrm{ff}$.

31 Rosenthal D. (Fn 29), Art. 10a DSG, N 111 und Art. 4 N77.

32 Rosenthal D. (Fn 29), Art. 4 N $67 \mathrm{ff}$. 